\title{
ON THE $\boldsymbol{H}$-FORCE NUMBER OF HAMILTONIAN GRAPHS AND CYCLE EXTENDABILITY
}

\author{
ERHARD HEXEL \\ Department of Mathematics \\ Technische Universität Ilmenau \\ Postfach 0565, D-98684 Ilmenau, Germany \\ e-mail: erhard.hexel@tu-ilmenau.de
}

\begin{abstract}
The $H$-force number $h(G)$ of a hamiltonian graph $G$ is the smallest cardinality of a set $A \subseteq V(G)$ such that each cycle containing all vertices of $A$ is hamiltonian. In this paper a lower and an upper bound of $h(G)$ is given. Such graphs, for which $h(G)$ assumes the lower bound are characterized by a cycle extendability property. The $H$-force number of hamiltonian graphs which are exactly 2-connected can be calculated by a decomposition formula.
\end{abstract}

Keywords: cycle, hamiltonian graph, $H$-force number, cycle extendability.

2010 Mathematics Subject Classification: 05C45.

\section{REFERENCES}

[1] A. Abueida and R. Sritharan, Cycle extendability and Hamiltonian cycles in chordal graph classes, SIAM J. Discrete Math. 20 (2006) 669-681. doi:10.1137/S0895480104441267

[2] G. Chen, R.J. Faudree, R.J. Gould and M.S. Jacobson, Cycle extendability of Hamiltonian interval graphs, SIAM J. Discrete Math. 20 (2006) 682-689. doi:10.1137/S0895480104441450

[3] R. Diestel, Graph Theory (Springer, Graduate Texts in Mathematics 173, 2005).

[4] I. Fabrici, E. Hexel and S. Jendrol', On vertices enforcing a Hamiltonian cycle, Discuss. Math. Graph Theory 33 (2013) 71-89. doi:10.7151/dmgt.1653

[5] G.R.T. Hendry, Extending cycles in graphs, Discrete Math. 85 (1990) 59-72. doi:10.1016/0012-365X(90)90163-C 
[6] T. Jiang, Planar Hamiltonian chordal graphs are cycle extendable, Discrete Math. 257 (2002) 441-444.

doi:10.1016/S0012-365X(02)00505-8

[7] R. Li, X. Zhang, S. Li, Q. Guo and Y. Guo, The H-force set of a hypertournament, Discrete Appl. Math. 169 (2014) 168-175.

doi:10.1016/j.dam.2013.12.020

[8] J.G. Oxley, Matroid Theory (Oxford University Press, Oxford, 1992).

[9] M. Timková, Enforced Hamiltonian cycles in generalized dodecahedra, Electron. J. Graph Theory Appl. 1 (2013) 77-88.

doi:10.5614/ejgta.2013.1.2.1

[10] X. Zhang, R. Li and S. Li, H-force sets of locally semicomplete digraphs, Discrete Appl. Math. 160 (2012) 2491-2496.

doi:10.1016/j.dam.2012.06.014

Received 27 July 2015

Revised 23 February 2016

Accepted 23 February 2016 